\title{
Real-time Forecasts of Tomorrow's Earthquakes in California: a New Mapping Tool
}

\author{
By Matt Gerstenberger ${ }^{1}$, Stefan Wiemer ${ }^{2}$ and Lucy Jones ${ }^{1}$
}

Open-File Report 2004-1390

2004

Any use of trade, firm, or product names is for descriptive purposes only and does not imply endorsement by the U.S. Government.

\section{U.S. DEPARMENT OF THE INTERIOR}

U.S. GEOLOGICAL SURVEY

${ }^{1}$ U.S. Geological Survey, 525 S. Wilson Ave. Pasadena, CA, 91106, USA

${ }^{2}$ Swiss Seismological Service ETH-Hoenggerberg/HPP-P7 CH-8093 Zurich, Switzerland 


\section{Abstract}

We have derived a multi-model approach to calculate time-dependent earthquake hazard resulting from earthquake clustering. This open-file report explains the theoretical background behind the approach, the specific details that are used in applying the method to California, as well as the statistical testing to validate the technique. We have implemented our algorithm as a real-time tool that has been automatically generating short-term hazard maps for California since May of 2002, at http://step.wr.usgs.gov

\section{Introduction}

Earthquake clustering, particularly in relationship to aftershocks, is an emperically defined, albeit not perfectly understood seismic phenomena. We have developed a real-time tool that combines and expands on previous research in earthquake clustering with current estimates of longterm hazard to create probabilistic forecasts. We combine an existing time-independent hazard model based on geological data and historical earthquakes with models describing time-dependent earthquake clustering. The result is a map giving the probability of strong shaking at any location in California within the next 24-hours, updated within minutes and available on the web at http://step.wr.usgs.gov. This report is a guide to understanding how this code works and how we have validated the results.

We expect the tool to foster increased awareness of seismic hazard in the general public, improved emergency response, and increased scientific understanding of short term seismic hazard. In the real time format, the maps provide:

- Assistance in immediate decisions by emergency planners and scientists related to the occurrence of a damaging earthquake after a large mainshock

- Information for city managers, etc., to assist in decisions about shutting down certain services and entering damaged buildings after a large mainshock.

- A continually updated source of information that is easily available to the general public and media.

Scientific advancements are in four main areas:

- Development of a real time probabilistic analysis tool 
- Increased understanding of spatial variability in aftershock sequences

- Implementation of a methodology for automating decisions related to model comparisons

- Establishment of a null hypothesis in time dependent and conditional probabilistic seismic hazard analysis.

Validation of a forecast model is a necessary part of its development. While other models have been developed to characterize short-term hazard [Kagan and Jackson, 2000], currently there is no established null hypothesis against which to test other models. By formulating our model as we have, we believe we have created an appropriate null hypothesis for testing other conditional probabilistic hazard and short-term seismicity related ideas (i.e. static and dynamic stress changes [Harris, 1998; Stein et al., 1992] accelerated moment release, [Bufe and Varnes, 1996] and quiescence before large aftershocks [Matsu'ura, 1986; Ogata, 1992]. We hope that the provision of this null hypothesis will encourage the development of other short-term models and also the participation of these models in model validation through statistical testing. This initiative is already well underway for southern California with the Regional Earthquake Likelihood Modeling (RELM) group of the Southern California Earthquake Center (http://www.relm.org). Much of our model has been developed with the goals of RELM in mind.

\section{Background}

The fundamental concept we used is an earthquake hazard map. These maps display the probability of exceeding a level of ground shaking in a specified time period, drawn from a model of earthquake occurrence and an estimate of the distribution of shaking that will result from each earthquake. Long term, probabilistic seismic hazard analysis (PSHA) and maps have been in use for a number of decades [Cornell, 1968; Frankel et al., 1996; Giardini, 1999] and are widely used in applications such as long term hazard mitigation through the design of appropriate building codes and the selection of relatively safe locations for building nuclear facilities. The maps display probabilities of exceeding a specified level of ground shaking over a long time period, typically on the order of 50 years.

We have expanded the concept of a hazard map by incorporating information about shortterm earthquake clustering. A simple schematic of how probabilities due to both foreshocks and aftershocks develop is illustrated in figure 1. Reasenberg and Jones [1989, 1990 \& 1994] developed a simple earthquake clustering model, based on the modified Omori law and the Gutenberg-Richter relationship, to describe this increase in probability of future events following any other event for time periods ranging from days to weeks. However, the model deals with time and magnitude only, not space. 


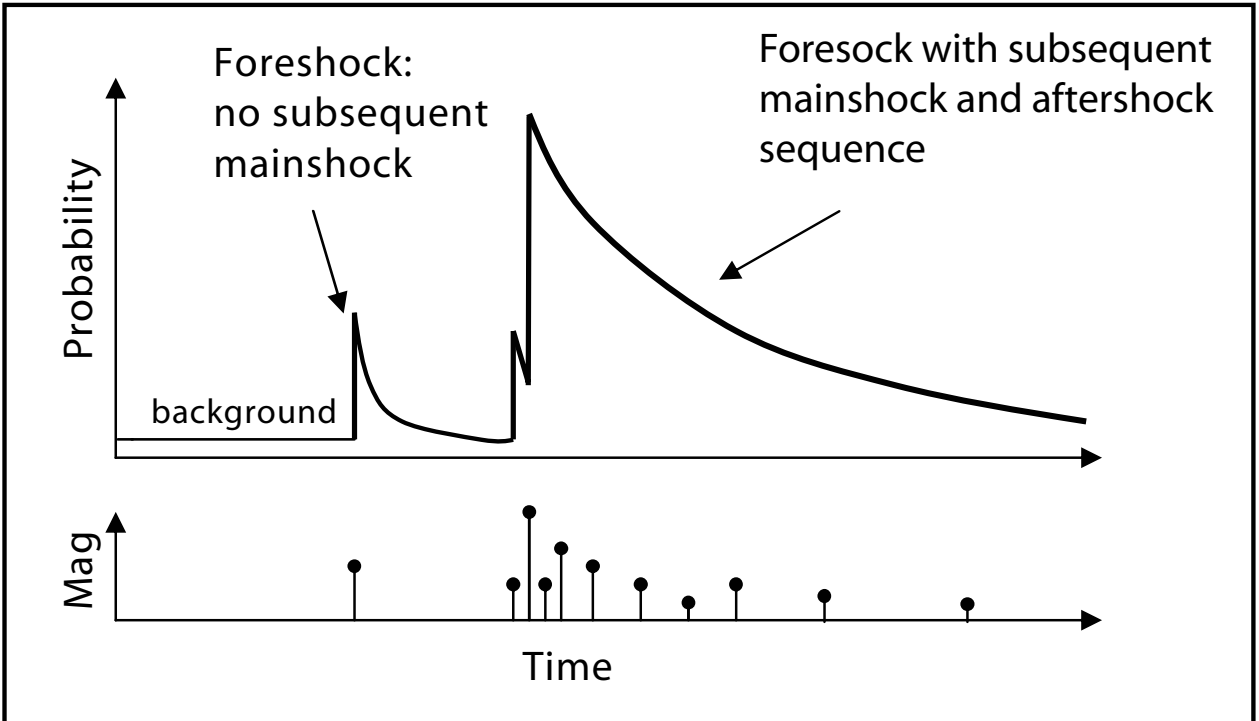

Figure 1: Schematic view of aftershocks and potential foreshocks and their effect on timedependent probabilities of future ground shaking. Bottom Axis: earthquakes of varying sizes including an aftershock sequence. Top axis: probabilistic hazard forecast from a simple clustering model based on the occurrence of each earthquake.

Reasenberg \& Jones (R\&J) made three assumptions about the distribution of aftershocks. First, they assumed that the aftershock magnitudes follow a Gutenberg-Richter relationship [Gutenberg and Richter, 1954], so that the number of aftershocks $(N)$ is proportional to the aftershock magnitude, $M$, as:

$$
N \propto 10^{b M}
$$

where $b$ parameterizes the ratio of small events to large events. Second, they assumed the rate of aftershocks varies with time as in the modified Omori Law [Utsu, 1995], so that the rate of aftershocks depends on the time since the mainshock, $t$, as:

$$
N \propto(t+c)^{-p}
$$

where $c$ and $p$ are empirically determined constants. Third, they assumed that the variation in aftershock rate with the mainshock magnitude, Mm, could be expressed through the GutenbergRichter relation, and thus:

$$
N \propto 10^{b M m}
$$

These three assumption were combined to give the rate of aftershocks $\lambda\left(t, M_{c}\right)$ larger than a magnitude threshold, $M_{C}$ and occurring at time t: 


$$
\begin{gathered}
\lambda(t, M)=\frac{10^{a^{\prime}+b\left(M_{m}-M\right)}}{(t+c)^{p}} \\
M \geq M_{c}
\end{gathered}
$$

From this rate, and assuming a non-homogeneous Poissonian occurrence of aftershocks, the probability of events occurring above a given magnitude and within a given time period can be calculated as follows:

$$
P=1-\exp \left[\int \lambda\left(t, M_{c}\right) \delta t\right]
$$

Thus the rate of aftershocks and therefore the probability of future aftershocks can be expressed with four parameters, $a^{\prime}, b, c$, and $p$. Reasenberg and Jones applied this model to California and determined these parameters for all sequences with mainshock magnitude $M \geq 5.0$ from 1932 to 1987. The generic aftershock sequence parameters have been defined as the mean of the approximately normal distributions of the constants from that dataset $\left(a_{g}^{\prime}=-1.67, b_{g}=.91, c_{g}=.05\right.$, $\left.p_{g}=1.08\right)$.

Figure 2 shows how forecasted rates of aftershocks from the generic model compare to observed rates from 1988-2002. This is an independent test which shows that the generic model is a reasonable first-order approximation of aftershock sequences occurring in the last 15 years. While the rates from the generic values do give a reasonable first estimate of aftershock hazard, they do not allow for variations in individual sequences by including any information specific to an individual aftershock sequence other than the mainshock size. As the sequences progresses, to best combine the information from the generic estimates and the sequence specific estimates, Reasenberg \& Jones proposed a technique based on Bayesian estimation.

\section{A Real-Time Model}

The method we describe in this report is an expansion of the Reasenberg and Jones model into a fully automated real time short term PSHA mapping tool. The maps combine information from long term hazard with short term hazard based on Reasenberg and Jones to produce a map showing the probability of exceeding a peak ground acceleration (PGA) level of .126 within a given time period, which is typically 24 hours.

We have expanded Reasenberg and Jones in several ways:

- The spatial distribution of the increased earthquake occurrence is computed: both the distribution of the overall hazard of the sequence around the mainshock fault, and the spatial variability within the sequence. 


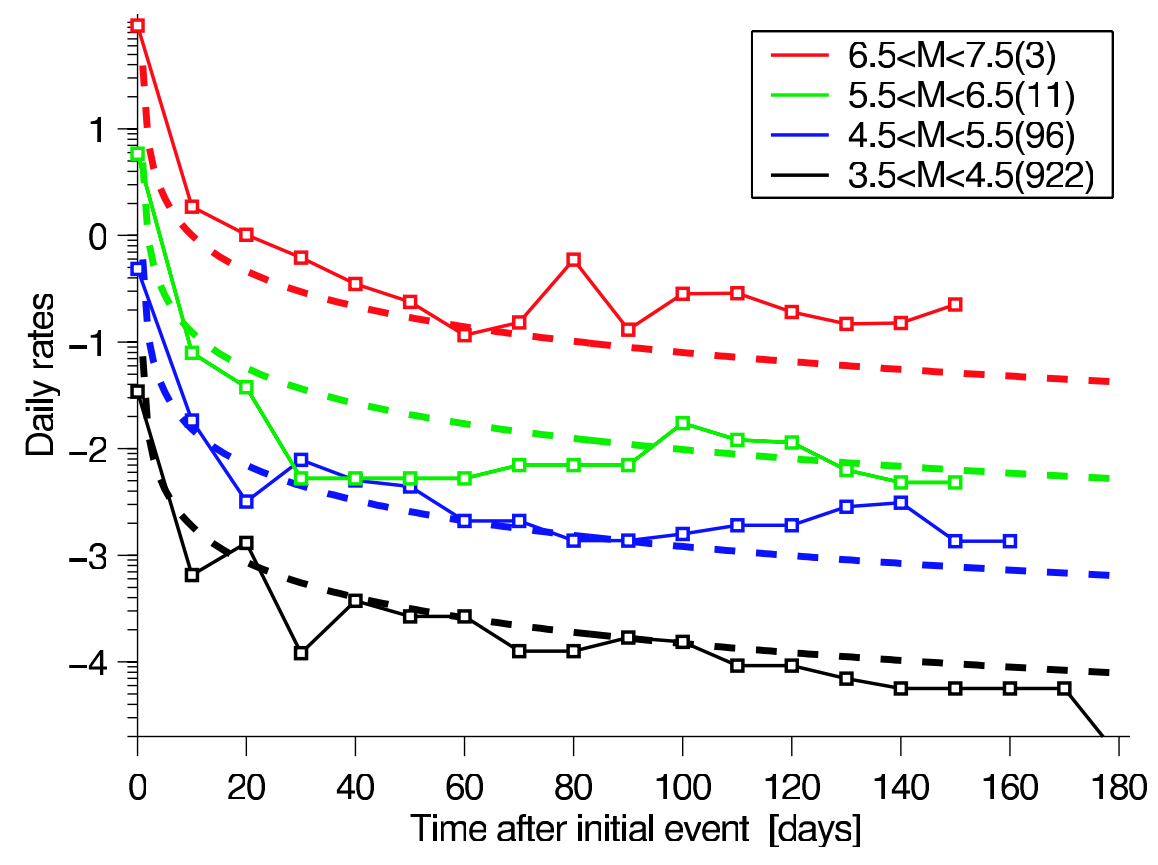

Figure 2: Calculated and observed rates of events $\geq M 4$ in 24-hour-long intervals following mainshocks occurring between 1988 and 2002 in southern California. Dashed lines show the rates forecasted by the generic California clustering model for the mainshock magnitude $(M)$ shown. Correspondingly, solid lines show the observed rates of $M \geq 4.0$ aftershocks for same time periods following mainshocks with magnitude $M \pm 0.5$. The aftershock zones are defined as the areas within one rupture length of the main shock epicenter.

- The combined hazard from multiple aftershock sequences and cascading sequences is included.

- A hazard assessment of seismic shaking from all possible magnitudes is calculated.

- The hazard is computed automatically for real-time use.

Our final forecast model is derived from several "model elements." These model elements, which we will define later, are earthquake rate forecasting techniques based on the previously mentioned seismicity parameters. Using the analogy of the logic tree approach often used in standard PSHA, our final forecast may be based on contributions from more than one model element at any given time. However, because of the need for real-time processing, the combining of the model elements must be based on an automated decision. Using the corrected Akaike Information Criterion (AICc) [Burnham and Anderson, 2002] we compare all model elements and derive a weighted and combined forecast of the future rate of events. 
This section gives a detailed description of the above process starting from recording the occurrence of a new event to the creation of a hazard map. In the following section, a detailed flow chart is presented.

\subsection{The Data}

The model is applied to the entire state of California. We collect information on earthquakes up to 0.5-degrees beyond the state boundaries. As implemented, maps are created for the whole state and, to provide more detail, for individual 3-degree squares across the region. Rate forecasts are calculated on a 0.05 -degree grid.

Real-time earthquake hypocenters and magnitudes are obtained from the California Integrated Seismic Network via QDDS, a U.S. Geological Survey application for distributing and receiving events in real time (ftp://clover.wr.usgs.gov/pub/QDDS/QDDS.html). As QDDS is a real-time application and collects earthquake data from a number of sources, duplicate earthquake locations and magnitudes can be received. Additionally, updated locations or magnitudes are sometimes distributed after the initial data have been received. Provisions have been made in the code to remove duplicate events and to include updated information relevant to a particular event. The initial locations from QDDS are computed automativally and can contain errors. The smoothing implicit in the gridding algorithm we describe below negates much of the effects of location errors, but it also limits the resolution of spatially variability that we are able to achieve.

\subsection{Spatial Definition of the Aftershock Zone}

For every recorded event, we must determine if the event should be modeled as an aftershock to some previous mainshock. The model defines aftershocks based only on space and time; therefore we must assign an aftershock zone to every event. Additionally, each time the code is executed, an aftershock zone is estimated or re-estimated for each event. Any single event is only allowed to be the aftershock to one mainshock. If the event falls within the spatial and temporal definition of the aftershock zones for more than one mainshock, the event will be assigned only to the largest mainshock.

Three types of aftershock zones are defined based on increasing complexity and amount of data required. Here we discuss the spatial component of the aftershock zone; the temporal component is explained in the 3.8.1.

- AftershockZone Type I: The least complex zone is based on a rupture length from the Wells and Coppersmith relationship for subsurface rupture [Wells and Coppersmith, 1994]: 
$\log (R L D)=-2.44+.58 * M$ where $R L D$ is the subsurface rupture length and $M$ is the moment magnitude. The zone is defined as the region within one rupture length of the epicenter of the event, resulting in a circular aftershock zone. This method requires only the event magnitude and location.

- AftershockZone Type II: When at least 100 aftershocks have been recorded within an $\boldsymbol{A f}$ tershockZone Type I, we use these events to define the aftershock zone based on the spatial distribution of the aftershocks themselves. We estimate the fault location in three steps:

1. Find the individual most northern, southern, eastern and western events (4 total events). Ignore the most extreme $1 \%$ events in all cases (e.g. ignore the $1 \%$ most northern events).

2. Using these four events, select the longest of the east-west or north-south dimension (length) and use the two events delineating this dimension to define the end-points of the fault.

3. Use these end-points to create a two segment segment fault that passes through the mainshock epicenter.

All events within one half of a fault length of the fault (as defined by Wells and Coppersmith) are considered aftershocks. A future improvement to Type II zones will be to allow for a twodimensional fault representation for thrust faults. An example of a Type II zone as calculated for Landers is shown in figure 3.

- AftershockZone Type III: Finally, if a fault rupture model has been calculated externally, we manually input this model and override the automatically calculated aftershock zones. The distance that the aftershock zone extends from the fault is part of the input model. Currently this last model is only two dimensional and is is only available for the largest mainshocks and could take a day or more after the mainshock before it is available. The reliance on the use of the surface expression of the fault limits the usability of this type of zone (e.g., a Type III zone was not applied to the Northridge earthquake because the surface fault will not represent the actual aftershock zone) and is an example of future work needed for the model. It should also be pointed out that the use of this externally input model is not an automated decision and requires input from an operator at the time of the fault model's implementation. An example of a Type III zone using the Wald and Heaton [1994] slip model is shown in the right panel of Figure 3. In this case the radius would be based on a seismologist's interpretation of the spatial extent of the seismicity. 


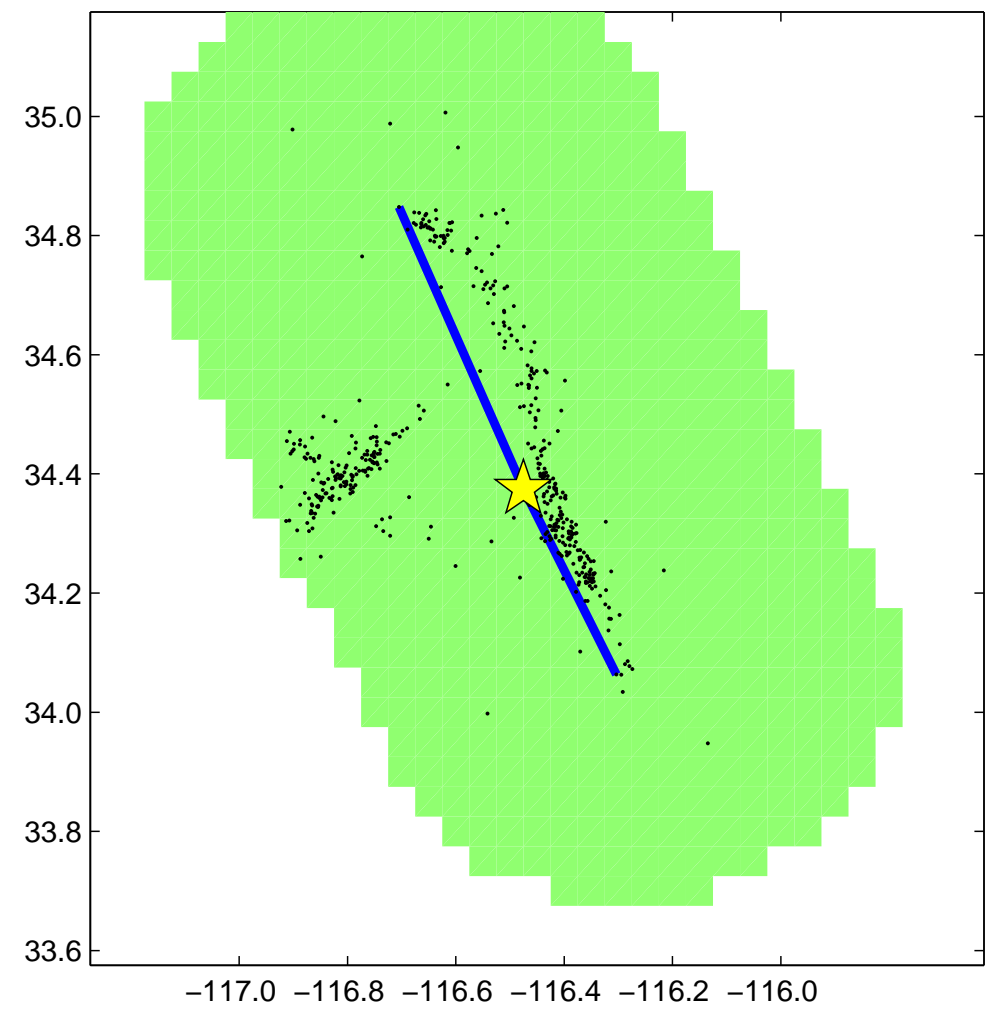

Figure 3: Figure showing the AftershockZone Type II as would have been calculated 24 hours following the Lander's mainshock (yellow star). The green area represents the total area of the aftershock zone; the blue is the estimated fault; and the black dots represent all aftershocks within the zone. 


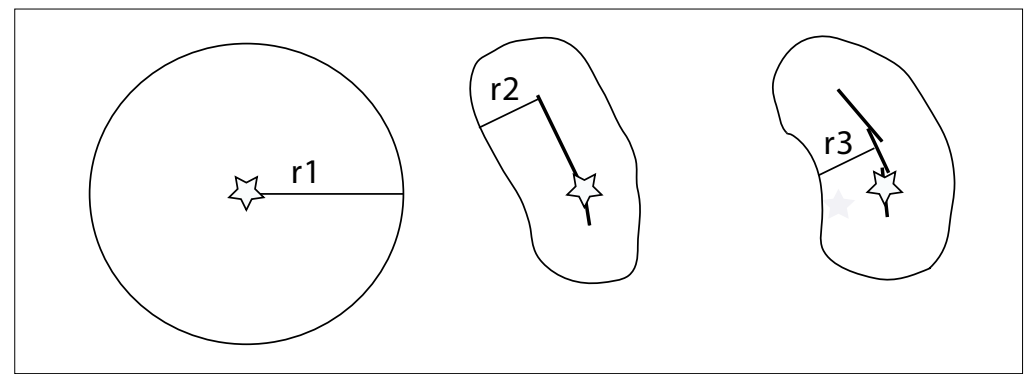

Figure 4: Cartoon of 3 possible aftershock zone models for Landers. Left-Type I: Simple model based on 1 Wells \& Coppersmith radius (r1) from mainshock epicenter. Center-Type II: Synthetic fault model keeping all events within $1 / 2$ of a Wells \& Coppersmith radius (r2). Right-Type III: External model based on the slip model of Wald and Heaton [1994] and keeping all events within given radius $r$.

\subsection{Parameter Estimation and Magnitude of Completeness}

To determine the spatial definition of the aftershock zone for a given mainshock as described above, we use all possible aftershocks regardless of magnitude. However, we must only use events larger than the the magnitude threshold of completeness, $M_{c}$ to estimate the parameters $(a, b, c \& p)$ for the rate forecast. $M_{c}$ is estimated using power law fitting techniques as described in [Wiemer and Wyss, 2000]. In all cases, we estimate $M_{c}$ for the entire aftershock sequence. When calculating spatially varying rates, we also estimate $M_{c}$ independently for the set of events for each grid node. All events smaller than $M_{c}$ are removed from the corresponding region. Both types of $M_{c}$ are re-estimated every time the code is initiated.

The parameters of an aftershock distribution are quite sensitive to the assumed magnitude of completeness, and some catalogs appear not to be quite complete at the level implied by a formal estimate of $M_{c}$. For this reason we take a somewhat conservative approach and assume the magnitude of completeness to be 0.2 units larger than the formal estimate. Furthermore, recent studies find that the magnitude of completeness is much larger immediately following an earthquake, because of the practical problem of identifying individual aftershocks when their records overlap. For this reason we estimate the model parameters using only aftershocks more than 0.2 days after the mainshock, provided that there are at least 100 such events. If not, we use all available events in the sequence.

\subsection{Spatial Distribution of the Aftershock Productivity}

The productivity constant, $a^{\prime}$ in Equation 3, defines the total rate of aftershocks for the whole aftershock zone. In order to create a map, the aftershock rate must be distributed appropriately 


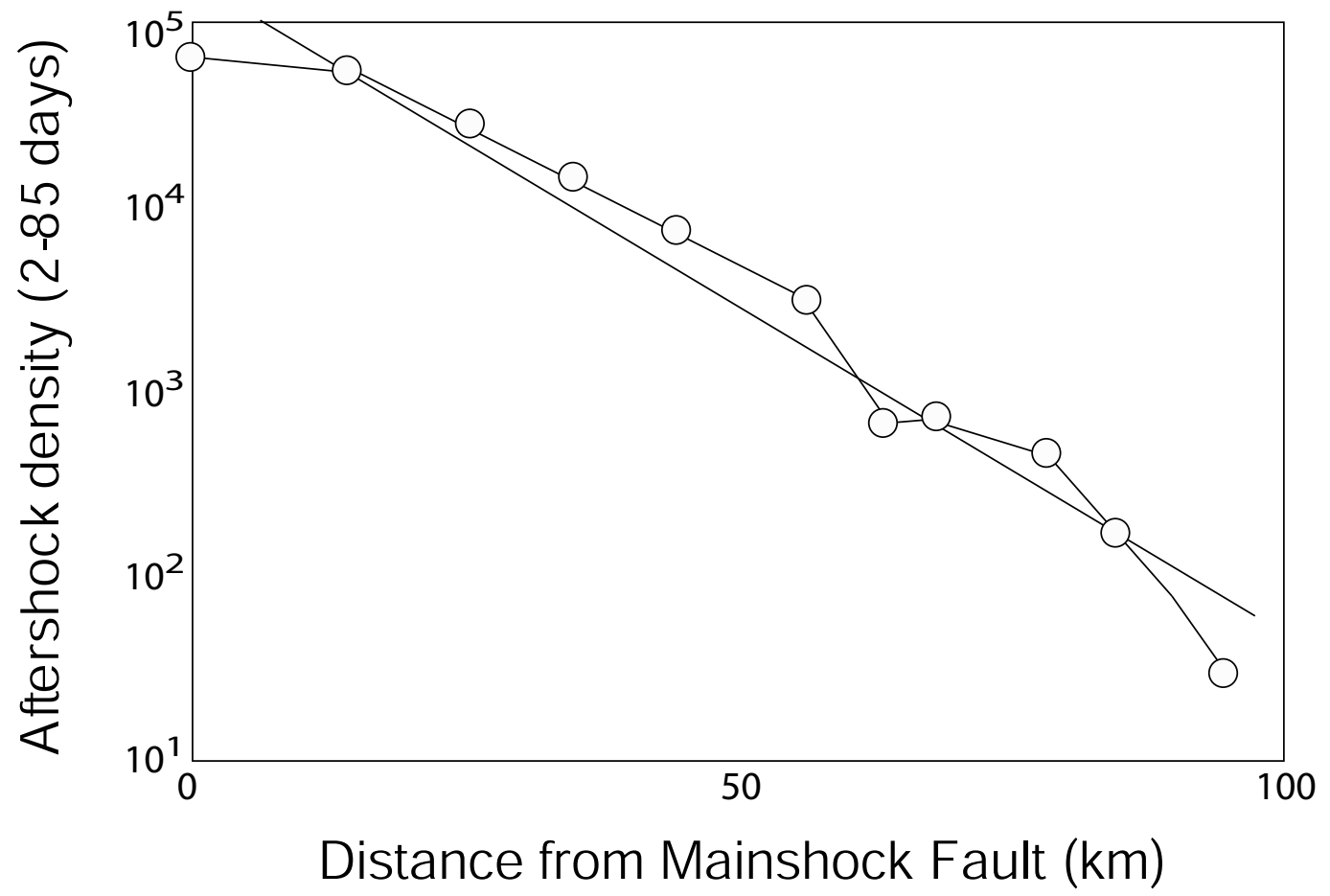

Figure 5: The rate of aftershocks as a function of distance from the mapped trace of the Landers earthquake rupture. The location of the fault trace is from Sieh et al., 1992. The locations of the aftershocks were determined with a three-dimensional velocity model by Hauksson (2000). The fit to the curve is equivalent to $\frac{1}{r^{1.8}}$

throughout the aftershock zone so as to retain the originally forecasted number of events. Aftershocks frequently occur at a distance away from the mainshock fault but a systematic study of the average distribution over many sequences has not yet been done. For the 1992 Landers earthquake, one of the few earthquakes for which accurate locations and systematic recording of aftershocks is available, the rate of aftershocks with distance from the fault approximately follows a $\frac{1}{r^{2}}$ distribution (Figure5).

Based on this understanding of California aftershock sequences, we taper the productivity by $r^{2}$, where $r$ is the distance from the fault; the minimum distance to any grid node is limited to $2.5 \mathrm{~km}$ (1/2 width of a grid cell) to avoid concentrating the rates inappropriately. In all cases no increase in productivity is forecasted outside of the aftershock zone boundary. This results in a circular smoothing around a point for a Type $I$ aftershock zone, and circular smoothing about a line (or line segments) for Type II and III zones. For the case of large events restricted to using a Type I aftershock zone, the smoothing is not entirely satisfactory and causes the rate increase to be smoothed over an unreasonably large area. However, this situation only occurs until enough events are available to estimate a fault, typically within the first hours of such sequences. An example 


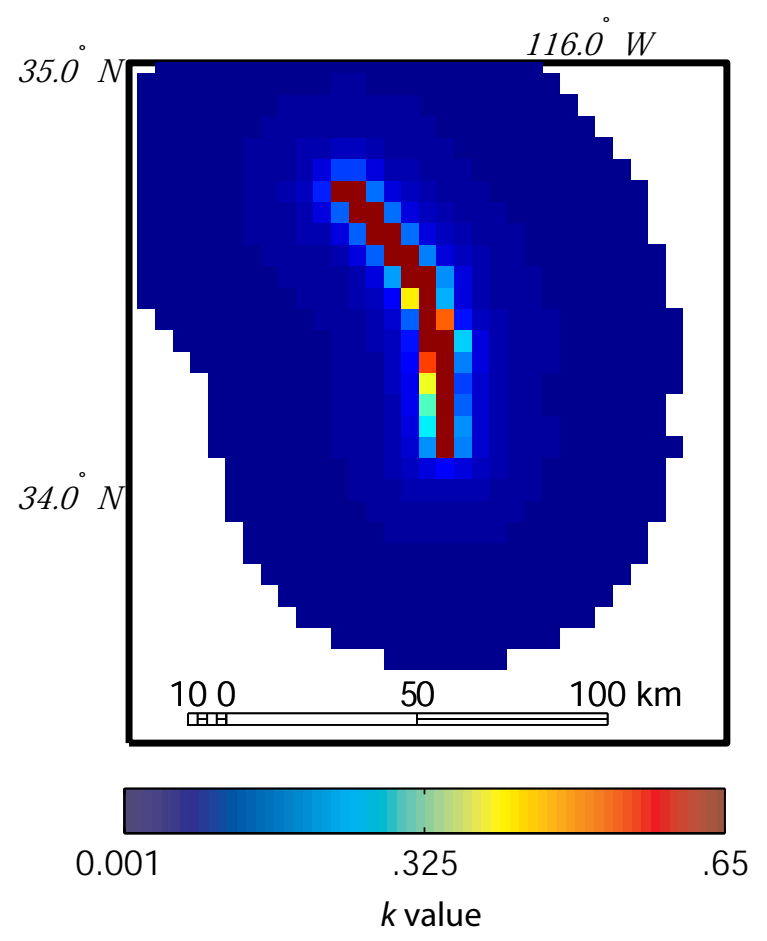

Figure 6: Plot of the smoothed $k$ value one day after the Landers mainshock; an external fault model has been input to define the aftershock zone. The aftershock zone width was defined using a $60 \mathrm{~km}$ half-width. The clear focusing of the rates along the fault is apparent in the figure.

of a smoothed aftershock rate (the 1992, $M_{w} 7.3$ Landers, California earthquake) is show in figure 6. The focusing of the rates in the 1 or 2 grid nodes (grid size: $5 \mathrm{~km} x 5 \mathrm{~km}$ ) either side of the fault is clear; the deepest red nodes correspond directly to the input fault model. Additionally, figure 7 shows the progression from a Type I aftershock zone to Type III for the Hector Mine aftershock sequence.

\subsection{Secondary Events}

An inherent limitation of the modified Omori law is that it assumes the occurrence of an individual aftershock has no effect on the rate of aftershock production of the overall sequence. Only the mainshock is allowed to excite an aftershock sequence; individual aftershocks do not contribute any rate changes of their own. In reality, secondary aftershock sequences are often observed [Ogata, 1999] with large aftershocks causing an increase in the aftershock rate in their immediate vicinity. All aftershocks appear to impart some change to the regional stress field, and larger aftershocks can cause a significant change in the rate of event production.

We account for these cascading aftershock sequences in our model by superimposing Omori 

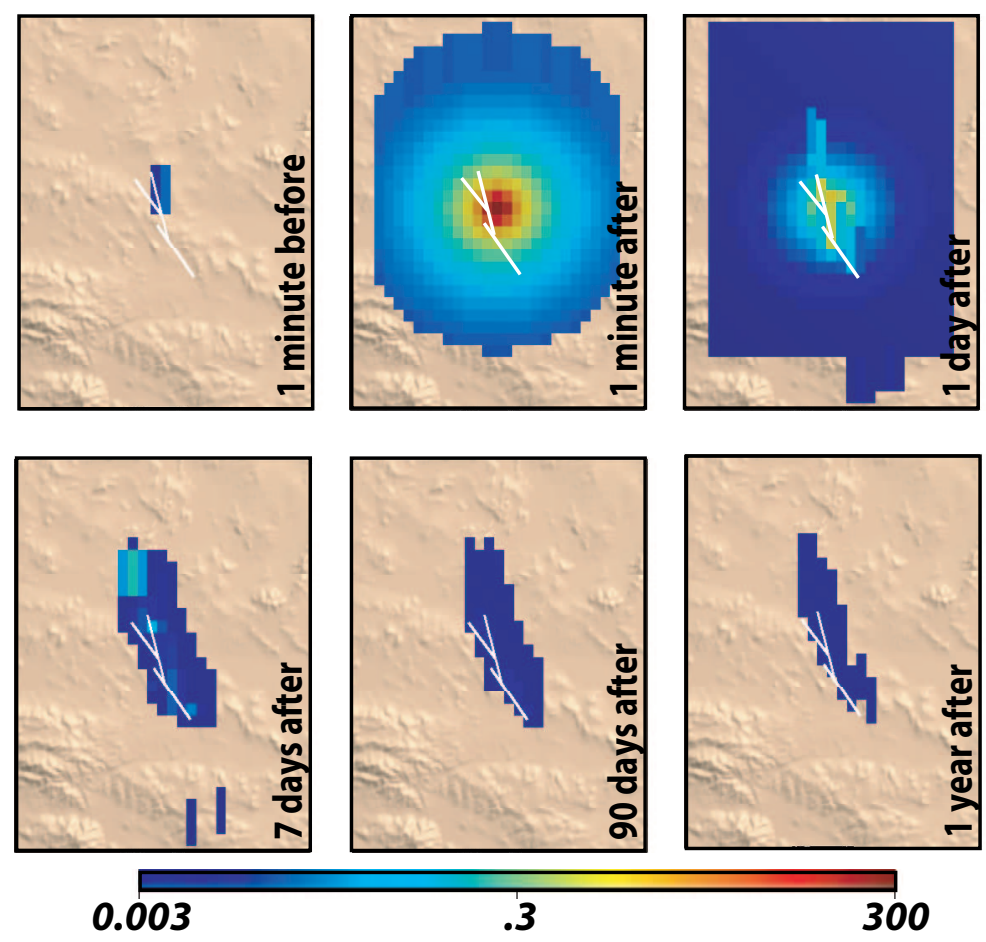

\section{Forecasted number of events above background}

Figure 7: Forecasted number of events $(M>4)$ above the background level for the Hector Mine aftershock sequence. Times are relative to the mainshock. The progression from a $100 \%$ generic model element using a Type $I$ aftershock sequence 1 minute and 1 day after the event to a combination of the three model elements is apparent 7 days after the event. The non-symmetric spatial variability in the rates at day one is due to secondary sequences. For simulation, a Type III (aftershock zone) fault based on Ji et.al. [2000] and an emperically determined radius is allowed one day after the mainshock. 
sequences. We allow every event within a sequence to play a part in two sets of rates; one set is the rates calculated by allowing the event to produce its own aftershock sequence and the other is from the recalculation of the rates of the initial mainshock after adding the secondary event to the sequence. Because of the difficulty in distinguishing aftershocks of secondary events from those of the primary event, all forecasts for secondary events use the generic parameters as well as Type I aftershock zones. It should be noted that events larger than the mainshock cannot be considered secondary events. In order to avoid double counting of these events, only the highest rate of forecasted events at any node is carried through to the hazard calculation.

Two non-trivial examples of how large secondary aftershocks are treated come from the $M_{w}$ 6.3 Big Bear event that followed approximately 3 hours after the 1992 Landers $M_{w} 7.3$ mainshock and the $M_{w} 7.1$ Hector Mine event that occurred in 1999. The Big Bear event occurred $35 \mathrm{~km}$ from the Landers epicenter, well within the aftershock zone of a M7.3 and was thus defined as a secondary aftershock; therefore, because Big Bear is secondary, all subsequent parameter calculations for it are restricted to the generic model. Seven years later, when the Hector Mine event occurred, the Landers aftershock zone would be based on an Type III aftershock zone (external fault model and radius) and would not encompass the Hector Mine mainshock location. Therefore

complexity beyond the generic and sequence specific model elements (figure 3) was available for use in calculating the Hector Mine seismicity parameters.

\subsection{Forecast Models Elements: Calculation of the Forecasted Rates of Events}

Once all aftershocks have been assigned, we must apply a model at each grid point to obtain the forecasted rate of events. The final rate forecast is derived from a combination of four model elements; each model element is calculated at each grid node whenever possible. The base model element is the:

- Background Model Element

This base is then added to a combination of the following time dependent elements:

- Generic California Model Element

- Sequence Specific Model Element

- Spatially Varying Model Element

The foundation of each time dependent element is a spatial distribution of seismicity parameters $a, b, c$, and $p$ which is used to calculate a rate forecast for the time period of interest, at each grid node, for each model element. 


\subsubsection{Background Model Element}

This model element contains the precomputed Poissonian and characteristic earthquake rate forecast for the time period of interest (daily) and does not contain time dependent components. We based the daily background rate on the long-term rate of the USGS National Hazard Maps (Frankel et al., 1996). First we interpolated the characteristic fault information onto our grid. Next, by assuming the same $b$ value as in the National Hazard Map background seismicity, we extrapolated this seismicity down to $M 4$ using the Gutenberg-Richter relationship. The characteristic fault information was interpolated to the given grid by: 1) projecting the fault to the surface, 2) distributing a large number of points over the surface projection of the fault, 3) counting the number of points that fell within a grid node, and 4) assigning the appropriate percentage of rates to each grid node, based on the percentage of overall points that fell within the node. This element will be updated in future versions as new Poissonian models are available.

\subsubsection{Generic California Model Element}

The generic California element calculates an earthquake forecast from the rate in Equation 3, using the generic California parameters. To map the forecast, we determine the spatial extent of the aftershock zone as described in section 3.2. We then smooth the seismic productivity as described in the section 3.4; otherwise the remaining parameters are the same for every location within the zone. This model element, as well as the background model element is always available.

\subsubsection{Sequence-Specific Model Element}

We attempt to determine sequence-specific aftershock parameters once we have recorded at least 100 aftershocks in a sequence above the $M_{c}$. With less than 100 events, not only is the uncertainty large, but typically no estimate of $p$ can be made by the code. We derive the sequence specific model element by determining the aftershock parameters, $a, b, p$, and $c$ from Equation 3 , that best model the recorded aftershocks. The parameters are estimated using maximum likelihood techniques originally developed by [Aki, 1965] and [Ogata, 1983a] and applied by [Reasenberg, 1994]. The parameter a is calculated by equating the rate parameter, $k$, in Omori's Law to the rate in the Gutenberg-Richter realtionship and solving for $a$ from $k$ and $b$. We spatially smooth the productivity of this model in the same manner as for the generic model. The fitting of the $c$ value is notoriously unstable, therefore we fix the parameter to a default value of .05. 


\begin{tabular}{|c|c|}
\hline Total number of events $>M_{c}$ & Radius \\
\hline$\leq 1000$ & $15 \mathrm{~km}$ \\
\hline$>1000 \& \leq 1500$ & $12 \mathrm{~km}$ \\
\hline$>1500 \& \leq 2000$ & $10 \mathrm{~km}$ \\
\hline$>2000$ & $7.5 \mathrm{~km}$ \\
\hline
\end{tabular}

Table 1: The radius used for the seismicity parameter calculation, dependent on the number of available events $>M_{c}$.

\subsubsection{Spatially Varying Model Element}

The final element, the spatially varying element, is based on spatial variations in the aftershock parameters. When examining aftershock sequences, researchers have seen strong spatial variations in seismicity parameters [Wiemer, 2002; Wiemer and Katsumata, 1999]. This spatial variability has been shown to significantly impact hazard [Wiemer, 2000]. While there is no current physical model to explain the observed variations in seismicity parameters, we have exploited these empirical observations in our probabilistic forecasts. This is the only element to contain non-radially symmetric information. Again, this element is not calculated until at least 100 events larger than $M_{c}$ have occurred.

We evaluate the spatial distribution of aftershock parameters using the technique described in Wiemer and Katsumata [1999]. We create a grid of evenly spaced nodes over the study area and take all events within a constant distance (described below) from each node to be used to describe the characteristics of the location. The $k, c, p$ and $b$ values are then calculated at each grid node using these events for the spatially varying model.

The spatial resolution of the seismicity parameters is limited by the number of available events, with $k, p$ and $c$ being less robust than $b$. We therefore select events using a radius that is dependent on the overall number of available events above $M_{c}$. We decrease the radius as the number of events increases in order to improve the spatial resolution; see table 1 for the radii used. We perform all calculations on a .05 degree grid. For nodes where it is not possible to estimate $p$ and $c$, or less than 100 events are available above $M_{c}$, no spatially varying parameters are estimated for the node and therefore the spatially varying model element adds no information, at that node, when the model elements are combined.

\subsection{Foreshocks: Forecasting Aftershocks Larger than their Mainshock}

So far we have focused our discussion on aftershocks. However, we are interested not only in hazard increases from events smaller than the mainshock (aftershocks), but also in the increased potential for larger earthquakes, e.g., foreshocks. Several authors [e.g., Reasenberg, 1999, Felzer, 
2003] have shown that the rate of mainshocks after foreshocks is consistent with an extrapolation of aftershock rates to larger magnitudes. Therefore we calculate the probability of subsequent events as small as magnitude 4 and up to magnitude 8 , for all magnitudes of mainshocks. While the probability of these larger events is quite small, it does represent a significant contribution to the hazard because larger events have a relatively large impact on the hazard forecast.

For every event we calculate the rate of forecasted events from a minimum size, $M_{\min }$, to an assumed maximum, $M_{\max }(4.0 \leq M<8)$. It is important to note that the inclusion of rates forecasted up to $M 8$ generally has a negligible effect on the final forecasted hazard; within any 24 hour period the probability of an $M 8$ event is not dominant over the hazard contributions from smaller events. One exception can be immediately after a large $M 7+$ event, when a $M 8$ event could contribute to the potential hazard for the initial hours/days after the mainshock. An interesting implication of this is that immediately after any large event, the probability of another large event is increased over what it was previous to the initial large event. For example, if a magnitude 7 event occurs in the Bombay Beach area of the San Andreas fault, there will immediately be an increased probability for another $M>7$ event to be triggered on the fault. However, less appealing examples are possible, such as the same thing occurring on a much smaller fault. This may not be entirely unreasonable, however; an example would be the Lander's fault which, prior to its latest rupture, was thought to be capable of producing only a magnitude 6.5 event but ruptured in a 7.3 event.

\subsection{Combining Model Elements}

At any give time it is possible that forecasted rates are being calculated based on 2 or 3 model elements (plus the background model element). Because of this, we need a selection routine that will automatically choose the best forecast. We wish to optimize the selection based on the amount of information available and do not wish to always force a single model element to be selected. Therefore we have implemented a scheme that uses the AICc (Akaiki Information Criterion) and AIC weighting [Burnham and Anderson, 2002]. This scheme allows for each model element to potentially contribute to the final forecast at any given node by summing the weighted contributions from each model element at each node. Because the background model element does not contain clustering information it is excluded from these calculations and only contributes where there is no current clustering activity.

The AIC provides a measure of fit, weighted by the number of free parameters, between a model and an estimate of the mechanism that explains the observed data. The corrected AIC includes terms for the amount of observed data, which becomes critical when sample sizes are small. The AICc is calculated as follows: 


$$
A I C_{C}=-2 \log (L)+2 K=\frac{2 K(K+1)}{n-K-1}
$$

where $K$ is the number of free parameters, $n$ is the sample size and $L$ is the likelihood function. The AICc gives us a relative score that is compared to the score from another model to obtain a relative performance measure.

Ogata [1983b] derived the following equation used to calculate the likelihood of an observed set of parameters from the modified Omori law, give an observation $N$ :

$$
L(k, c, p)=N \ln k-p \sum_{1}^{N} \ln \lambda(t+c)-k A(c, p)
$$

where $N$ is the number of events, $\lambda$ is the forecasted rate of events, $t$ is the time of the event, and $k$, $c$ and $p$ are the parameters of the modified Omori law. $A(c, p)$ is calculated as follows:

$$
\begin{gathered}
A(c, p)=\ln (T+c)-\ln (S+c) \text { for } \mathrm{p}=1 . \\
A(c, p)=\left\{(T+c)^{1-p}-(S+c)^{1-p}\right\}(1-p)^{-1} \text { for } \mathrm{p} \neq 1 .
\end{gathered}
$$

Where $S$ is the time of the first event and $T$ is the time of the last event.

We calculate an AICc value for every grid node and assume the sample size is the number of observed events for the grid node. The number of free parameters is defined as: 0 for the generic model, 0 for the sequence specific model, and 3 for the spatially varying model with fixed $c$. These numbers are an estimate based on the fact that we calculate an AICc at every grid node; at an individual grid node, there are effectively no free parameters for either the generic or the sequence specific model. After we have calculated an AICc score for each model at each grid node, we calculate a weight for each model at each grid node by calculating the Akaike Weights following the method described by Burnham and Anderson:

$$
w_{i}=\frac{\exp \left(-\frac{1}{2} \Delta A I C c_{i}\right)}{\sum_{r=1}^{R} \exp \left(-\frac{1}{2} \Delta A I C c_{r}\right)}
$$

where $R$ is the number of models and $i$ represents a single model. We then weight each model's forecast appropriately and sum them to obtain a composite forecast for each node. We make no restrictions on the variability of the weighting from node to node and allow the element that best describes the data at each individual node to be weighted the most heavily.

\subsubsection{Combining the Short Term Hazard with the Background Model Element}

We combine the weighted rate forecast with the background model element rates. It is possible to have a weighted rate forecast from more than one aftershock sequence for any grid node at a 
single time; therefore we add only the highest forecasted rates at any node to the background. Additionally, we only add the rate of forecasted events if it is greater than the background rate for the corresponding node. The steps we follow are: 1) at each node, determine the highest short-term forecasted rate from all active sequences for that node, 2) subtract the background forecast from our short-term forecast, 3 ) set any negative rate to 0,4 ) add these new rates to the background.

The decision on when to decide a sequence is no longer active and to no longer continue to forecast rates based on it is made by comparing the total number of forecasted events $(M>5)$ to the forecasted number in the background. If this total number drops below the background level, the sequence is terminated. Because we terminate a sequence when its rates reach the background level, this method assumes that the background rates are represented in the short term forecasts.

\subsection{Calculation of Probabilities of Ground Shaking Exceedance.}

Once the forecasted rates of events for each sequence have been estimated, we then calculate the hazard. The hazard calculation follows the standard practices of PSHA [Cornell, 1968] and for simplicity we will not discuss it in this report.

Because we wished to present the results to the general public in an accessible form we follow the example of ShakeMaps and use Modified Mercalli Intensity [Wood and Neumann, 1931] for the final presentation. Level VI was chosen because it is when objects are thrown off shelves so that the public considers it damaging. To get from PGA to MMI we use the relationship from Wald, et. al., [1999a]:

$$
I_{m m}=3.66 \log (P G A)-1.66
$$

and derive PGA .126 as a proxy for MMI VI. Thus, we calculate a mean probabilistic seismic hazard (PSHA) map that gives the log probability of exceeding PGA .126 within a given time period (24 hours).

Currently we treat all events as point sources for the hazard calculations. The limitation of this is that source-receiver distances may not be adequately represented for the attenuation calculations. As this is a common problem for grid-based hazard algorithms, we will be working with RELM and OpenSHA for an acceptable solution.

For events of magnitude 5.5 and greater we have chosen to apply the Boore, Joyner and Fumal attenuation relationship [Boore et al., 1997] with the standard PGA coefficients and assuming a rock site. However, for the relatively low level of hazard that we are interested in, events as small as magnitude 4 can potentially contribute to hazard and we wish to include them in the calculations. Therefore, for events between magnitudes 4 and 5 we use a set of coefficients derived from small California mainshocks [Quitoriano, 2003] and apply a linear smoothing between the two from 


\begin{tabular}{|c|c|c|c|c|c|c|c|c|c|}
\hline & $b 1$ & $b 2$ & $b 3$ & $b 5$ & $b v$ & $h$ & $V a$ & $V s$ & $\sigma$ \\
\hline \hline$M<5.5$ & 2.4066 & 1.3171 & 0 & -1.757 & -0.473 & 6 & 760 & 620 & .52 \\
\hline$M \geq 5.5$ & -0.313 & 0.527 & 0 & -0.778 & -0.371 & 5.57 & 1396 & 620 & .52 \\
\hline
\end{tabular}

Table 2: coefficients used in the BJF attenuation relationship [Quitoriano, 2003] .

magnitude 5 to 5.5 (table 2).

\subsection{Processing Flow Chart}

The following section describes the steps that are followed each time the code is triggered. The current and tested version of the code is written primarily as a suite of Matlab [reference] functions, with the reliance on several Linux scripts and $\mathrm{C}$ language programs for controlling the real time operations. Rigorous validation of the code has been limited at the time of publication; however the next generation of the code is currently being written in Java to allow easy interfacing with validated methods provided by OpenSHA [Field, et. al., 2003]. Translation of the code into Java will also allow for full validation of the code by comparing results of the Java implementation to those of the Matlab version.

1. Every $N$ minutes launch the hazard code. Currently the code takes approximately 10 minutes to create either a southern or northern California map and is re-launched every 15 minutes.

2. Load scratch files (Sec. 13.(a)) that contain the details from the previous execution of the code. Specifics included in the scratch files are as follows:

(a) all events from the previous code execution that generated a forecasted rate of events ( $M \geq 5$ only) anywhere greater than the background rate for the same location: henceforth referred to as previous events.

(b) all aftershocks which were previously assigned to a mainshock (previous event) and flagged as such.

(c) the seismicity parameters $(k, b, c \& p)$ that were assigned to previous events/sequences (overall sequence including each grid node) in the last code execution.

3. Load all events delivered by QDDS since the previous execution of the code. Perform error checking to remove duplicate events and correct updated events.

4. Load the gridded background daily rate map (background model element). 
5. Update the aftershock zone for each previous event, in chronological order, and locate its new aftershocks:

(a) If an externally calculated fault model is available, it is loaded here to define a Type III aftershock zone. Find and flag all new events within the aftershock zone. If a new event is:

i. smaller than the previous event, flag the new event as a secondary event.

ii. larger than the previous event, restrict the previous event to using its last calculated seismicity parameters (loaded in step 2.(c)) in all future calculations.

(b) If an external model is not available, use a Type I aftershock zone (even if a Type II zone was used in the previous code execution).

i. Follow the rules of 5.(a) for collecting and flagging of aftershocks.

(c) If 100 or more aftershocks were defined in the previous step (5.b), estimate the aftershock zone using the spatial distribution of these events: a Type II aftershock zone.

i. Remove flags of all events defined in the previous step and locate and flag all events within the Type II zone.

(d) Define the aftershock zone grid:

i. Create a .05 degree grid over the aftershock zone where the grid points are aligned with the background model grid.

6. In chronological order, define the aftershock zone for all new events and collect any aftershocks by performing the following steps for each new event:

(a) For all primary new events (events not already flagged as aftershocks):

i. Using the rules of step 5, define the appropriate aftershock zone and collect all aftershocks within the zone that are not assigned to a prior mainshock.

(b) For all new events that have been flagged as secondary (aftershocks) in step 5 or step 6.(a):

i. Define a Type I aftershock zone but do not collect aftershocks

(c) New events that are M1.9 or below:

i. Events that are not flagged as secondary are ignored (i.e., they are never treated as a mainshock that initiates its own aftershock sequence).

ii. Events flagged as secondary are used for later calculations of the assigned aftershock zone. 
7. Estimating the overall $M_{c}$ for an aftershock sequence:

(a) If more than 100 events have occurred past 2 days in a sequence, flag all events before .2 days to be ignored in the $M_{c}$ and parameter calculations. (Note: in the case that this results in too few events to be used in subsequent calculations (8.b.i \& 8.c.i), these events are not added back in).

(b) Calculate the $M_{c}$ for each aftershock sequence and flag all events below the estimated magnitude so they are ignored in later steps. (Note: the spatially varying model element requires an additional completeness estimation. See step 8(c).iii.)

8. The forecast model elements. For each sequence calculate a grid of seismicity parameters and rates based on each of the three model elements, if possible:

(a) Generic California model element (Note: a secondary aftershock is limited to using only this element):

i. Calculate a generic $k$ value based on the generic $a$ and $b$ values and the forecast magnitude range of interest.

ii. Spatially smooth $k$ over the grid and assign a smoothed value to each grid cell.

iii. Assign the generic $b, c$ and $p$ values to all cells.

iv. Calculate a forecasted rate of events, for the time period of interest, for each node based on Equation 3.

(b) Sequence specific model element.

i. Only calculate if more than 100 events above $M_{c}$ have been recorded in the sequence

ii. Estimate seismicity parameters $b, p$ and $k$ for the sequence. Fix $c$ to .05 .

A. If the $p$ value is undetermined, increase $M_{c}$ by .1 and flag (and ignore) all events below this magnitude. Repeat until a $p$ value can be estimated or until fewer than 100 events remain that are greater than $M_{c}$. In the latter case, the sequence specific model element will not be not used.

iii. Spatially smooth $k$ over the grid and assign a smoothed value to each grid cell.

iv. Assign the estimated $b, p$ and $c$ values to each cell.

v. Calculate a forecasted rate of events, for the time period of interest, for each node based on Equation 3.

(c) Spatially varying model element. 
i. Only calculate if more than 100 events above $M_{c}$ have been recorded in the sequence

ii. Select events within the given radius from each grid node. The radius definition is given in table 1.

iii. Calculate $M_{c}$ for each grid node, using only the events selected in the previous step. Flag all events with $M<M_{c}$ so they are ignored in the next step.

iv. Estimate $k, p$ and $b$ for each grid node; $c$ is fixed to .05. Ignore grid nodes with fewer than 100 events remaining after the previous step

v. Calculate a forecasted rate of events, for the time period of interest, for each node based on Equation 3.

9. Model element selection and composite model creation.

(a) For each sequence, for the time since the mainshock to the current time, find the observed number of events in each magnitude bin within each grid cell.

(b) Calculate the likelihood for each model element for each grid cell based on the $k, c$ and $p$ values and the observed number of events.

(c) Calculate the Akaike Information Criterion for each grid cell for each model element.

(d) Using Akaike Weighting calculate a weight for each model element for each grid cell.

(e) Create a composite forecast for each grid cell by applying the model weights to each respective rate forecast and summing them.

10. Maximum rate calculation.

(a) For every grid cell on the map find the maximum forecasted rate based on the forecasts from all mainshocks, secondary events and the background; retain only the highest forecasted rate at each node.

11. Create a total forecast map.

(a) For each grid cell add the number of forecasted events, above the background level, to the background. This is done individually for each magnitude bin.

12. Calculate the hazard map using the background map grid.

(a) Calculate the forecasted ground acceleration at each node using the composite forecast. This is done using the Boore, Joyner, Fumal attenuation relationship (see previous section for a description of the method). 
(b) Calculate the probability of exceeding $.126 \mathrm{~g}$ for each grid cell (see previous section for a description of the method).

13. Write scratch files.

(a) For each grid cell save all events that initiate a forecasted rate of events $(M>5)$ greater than the background forecast for the same cell. Also retain, for the overall sequence and for each grid node, all seismicity parameters $(k, b, c \& p)$. Finally, all aftershocks to the event are retained.

14. Generate the hazard map image

(a) Currently we create a single map for all of California. The map is automatically updated twice an hour: once every 30 minutes. An example map created on August 2, 2004 is shown in figure 8.

\section{Model Validation}

Main [1999] introduced a 4 level sliding scale of earthquake prediction ranging from time-independent hazard (standard probabilistic seismic hazard maps), to deterministic prediction (where the location, time and size of an earthquake are reliably known ahead of time). The model we evaluate here falls under Main's second level category: time-dependent hazard. That is to say, we propose a degree of predictability based on the modified Omori law [Utsu, et. al., 1995] and the Gutenberg-Richter relationship (Gutenberg and Richter, 1954] but do not purport to know any particular features of an event that would upgrade the information to a higher level prediction. For the purpose of this report, we will refer to our predictions as forecasts.

The aims of statistical testing are to verify a particular forecast model and to ensure that its results are repeatable and not purely random. This is necessary if one wishes to understand if their model performs as desired or exhibits any unexpected behavior. Via rigorous statistical testing, the trap of unwittingly conditioning the data or testing to serve the modelers needs can be avoided [Console, 2001]. Typically, and in our case, any sort of forecast or prediction has ultimate goals in providing information to the public. This entails a responsibility of accuracy in the information that can only be ensured through statistical testing and model validation.

In our case, model validation includes tests providing two distinctly different types of information. First we wish to test our model against an established null hypothesis and second we seek to see if any statement can be made about the consistency of our model with observed data. The 


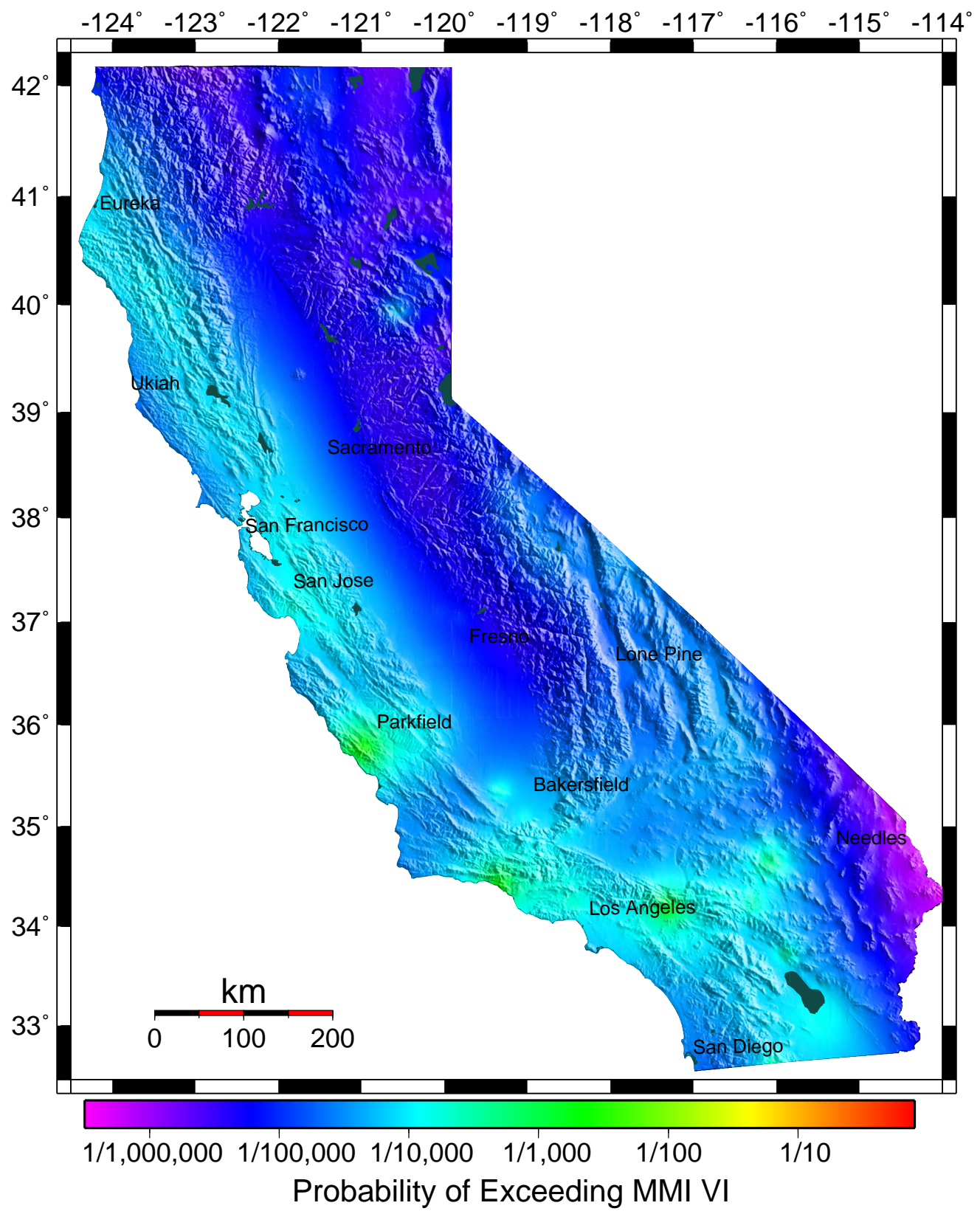

Figure 8: Hazard map calculated on Aug. 2nd, 2004. 
difficulty in the first test is finding an appropriate null hypothesis (for which we use the following definition: an established and accepted model of the same genre as our model). In the case of time-dependent hazard, there appears not to be an established null hypothesis. While this leaves the stage open for us to provide one, it leaves us with somewhat unsatisfactory choices for use in our testing.

We have chosen as our first null, our background model, the Poissonian USGS hazard map for California [Frankel, et al, 1997]. As this model does not include any clustering, and in fact specifically excludes hazard due to aftershocks, it would appear obvious that our clustering model should easily beat it. However, we can make no quantitative statement before performing a test. It is entirely possible that an inappropriate time-dependent model could fare worse than a timeindependent model when tested against real data. However, any reasonable model that includes clustering should be favorable.

The next logical null hypothesis is the Reasenberg and Jones model. Unfortunately, due to a lack of any sort of spatial information, their model as published is not testable directly against our model. However with the application of a few assumptions we can create a model that is. We test against the generic California model as well as the generic California model combined with parameters estimated for individual sequences (previously defined as a sequence specific model).

In the parameter optimization test we create forecasts based on the different parameter combinations we wish to explore and compare them to earthquake data a posteriori. This allows us to select the best parameter set based on current knowledge. We describe these assumptions in a later section.

\subsection{Testing Methodology}

In cooperation with members of the RELM group, we have participated in the development of a suite of statistical tests to be used in the testing of earthquake forecasts [Schorlemmer, et al, in prep]. In this section we describe the two RELM tests that we have used in this study. The foundation of both tests that we conduct is the likelihood. A widely used statistic, the likelihood is somewhat similar to the residual sum of squares method in that it gives a value related to the consistency of a model and its parameters to observations. However, instead of giving a norm of residuals between the observations and the model it gives the probability density of making those particular observations given the model parameters. A likelihood, therefore, gives an indication of how consistent a model is with the data.

The two tests we discuss here are:

- Consistency test: likelihoods calculated using instrumental data and simulations. In this test we investigate how well our hypothesis fits the data and if we can reject the hypothesis with 
the given significance. This test is designed to answer the question of how well does our hypothesis do at forecasting real data.

- Ratio test: likelihoods calculated using instrumental data and simulations; this test investigates if one hypothesis can be rejected in favor of another with the given significance. An important question in the design of our hypothesis is whether the addition of complexity (e.g. spatial variations in the $b$ value) improves the forecasts over a more simple hypothesis. The ratio test is designed to answer this question.

We test the forecasted rates of events rather than the forecasted probabilities of ground motion, for two reasons. Calculating forecasted ground motions adds a large amount of uncertainty (due to the attenuation relationship, site and source characteristics) to the forecast and we do not wish to have this uncertainty obscure our actual rate forecast. Additionally, the data that would be necessary for performing retrospective tests of ground motion is very limited.

An unbiased test of forecasted rates would be to calculate a rate forecast for a future time, wait for this time to pass and then test how the model did by calculating a likelihood. Unfortunately it can take a long time before enough events have occurred so that one can obtain a strong result. We approach this problem by using historical data and performing retrospective tests. However, as part of the RELM group, prospective tests will be initiated in the near future.

The likelihood value is a measure of the probability that a similar result would be obtained under a given hypothesis, and for that we need a probability distribution of earthquake numbers in a given region. Many seismological studies assume a Poisson processs, in which the earthquake rate is constant in time, independent of past events. However, Michael [1997] and others question its validity for earthquake occurrence, and the Poisson process is inconsistent with clustering. Nevertheless, for short enough time intervals the earthquake rate may not vary too much, and the Poisson process may be a good approximation as long as the rate is re-evaluated for each time interval. A generalized Poisson process for which the basic rate depends on time is known as a non-stationary Poisson process.

A simple likelihood alone is not sufficient; if we wish to make a statement about the consistency of our model with the data or when comparing our model to another model with the goal of rejecting, we must be able to attach a significance to this statement. We must be able to say that the results we have obtained are not simply due to random chance and that in another situation the results would be similar [Joliffe and Stefanson, 2003]. We therefore need a probability distribution of likelihood scores. Unfortunately, an analytical derivation of this is not practical and we must generate it through simulations.

To simulate catalogs, we must first create our forecast for the test period. The forecast must 
include the expected number of events for each magnitude $(4<M<8)$ and location being tested. We then assume there is a random probability governed by the Poisson distribution for a number of events to occur in each magnitude-space bin that is other than the forecasted number and draw a simulated number of events for each magnitude and location. This is done for two reasons: 1) because we wish to gain insight into how our model is performing and do not wish to perform a binary test where a forecasted event is $100 \%$ correct or $100 \%$ wrong; and 2) because our model provides a probability of events occurring and not an exact whole number of forecasted events (e.g., for the time period of interest we might forecast .1 events for a given magnitude in a particular grid cell). This allows us to calculate a distribution of earthquakes that is theoretically consistent with the model but that also allows for the random Poissonian scatter that is implied in the model. For each bin we calculate many simulations (typically 1,000) and obtain a likelihood for each, resulting in a distribution of likelihoods.

If the likelihood of the observed data occurs in the lower 5\% tail of this distribution, then the observation is unlikely to occur in the model, and therefore the model can be rejected with a significance of $5 \%$. Thus there is less than a $5 \%$ chance that we would reject a true hypothesis.

In our simplest test, a consistency test, we simulate earthquakes that obey the assumptions of the hypothesis being tested, calculate the likelihood scores for these simulations and then compare them to the likelihood of the observed data. If the tested hypothesis is consistent with the observed data, the likelihood of the observed data should not fall among the outliers of the distribution of the simulated likelihoods but should be clearly within the distribution.

The procedure for the ratio tests is similar to the method used for the consistency test. One important difference is that we calculate the ratio of the likelihood of the null hypothesis over the likelihood of the alternative hypothesis. This allows us to investigate whether we can reject the null hypothesis in favor of the alternative hypothesis, and if so attach a significance to the result. Because we simulate earthquakes based on the null hypothesis only, there are just two possible outcomes: rejection of the null hypothesis, or no rejection of the null hypothesis.

The ratio test only compares the two hypotheses; it does not test whether either is truly consistent with the data. If the null hypothesis is rejected, it may happen that both hypotheses are consistent (by the consistency test above), or that only the alternative hypothesis is consistent, or that both are inconsistent.

\subsubsection{Test Calculations}

In testing a forecast, we require the forecast to be specified as an earthquake rate per area, time and magnitude bin. The simplest way to test the model is to use a grid based test. Therefore, to remain consistent with the resolution of our forecast, we test on the same grid as we use to initially create the forecast, a $.05^{\circ} \mathrm{X} .05^{\circ}$ grid. In order to reduce the computational expense, we 
currently perform only one test per day. This unfortunately limits the resolution of our results. In the initial time period after a large mainshock, the production of aftershocks decreases very quickly; a substantial reduction in earthquake rate will occur within the first 24 hours. Due to using large time steps we integrate this change over a longer period and potentially miss testing the ability of our model to correctly forecast these temporal changes. Ideally we would test more frequently or only at the time of event occurrence. However due to the computational limitations mentioned above we were unable to do so in this study.

We denote each bin $b$ as a subset of total set of bins $\mathcal{B}$ :

$$
\mathcal{B}:=\left\{b_{1}, b_{2}, \ldots, b_{n}\right\}, n=|\mathcal{B}|
$$

where $n$ is the number of bins $b_{i}$ in the set $\mathcal{B}$. For each bin, we have a forecasted rate of events $\lambda_{i}$ with the total forecasted rate defined as:

$$
\Lambda=\left(\begin{array}{c}
\lambda_{1} \\
\lambda_{2} \\
\vdots \\
\lambda_{n}
\end{array}\right), \lambda_{i}:=\lambda_{i}\left(b_{i}\right), b_{i} \in \mathcal{B}
$$

We bin the observed events, $\omega_{i}$, in the same manner as above resulting in:

$$
\Omega=\left(\begin{array}{c}
\omega_{1} \\
\omega_{2} \\
\vdots \\
\omega_{n}
\end{array}\right), \omega_{i}=\omega_{i}\left(b_{i}\right), b_{i} \in \mathcal{B}
$$

As stated previously, we assume that for the time period of each test, earthquakes are independent of each other. This allows us to calculate the probability, given the forecasted rate $\lambda$, of observing $\omega$ events per bin based on the Poissonian probability:

$$
p(\omega \mid \lambda)=\frac{\lambda^{\omega}}{\omega !} e^{-\lambda}
$$

The $\log$-likelihood $L$ for observing $\omega$ earthquakes from a given forecasted rate $\lambda$ is defined as the logarithm of the probability $p(\omega \mid \lambda)$ :

$$
L(\omega \mid \lambda)=\log p(\omega \mid \lambda)=-\lambda+\omega \log \lambda-\log \omega !
$$

for each individual bin. From this we can obtain the total log-likelihood for all bins by calculating 
the log of the product of all probabilities (the joint probability):

$$
L(\Omega \mid \Lambda)=\sum_{i=1}^{n} L\left(\omega_{i} \mid \lambda_{i}\right)=\sum_{i=1}^{n}-\lambda_{i}+\omega_{i} \log \lambda_{i}-\log \omega_{i} !
$$

This is the log-likelihood we use for the consistency test. To compare two models, we must compute the log-likelihood-ratio:

$$
R=L\left(\Omega \mid \Lambda^{0}\right)-L\left(\Omega \mid \Lambda^{1}\right)=L^{0}-L^{1}
$$

where $\Lambda^{0}$ is the forecasted rates of hypothesis $\mathrm{H}^{0}$ and $\Lambda^{1}$ is the forecasted rates of hypothesis $\mathrm{H}^{1}$. $L^{0}$ and $L^{1}$ are the respective hypothesis joint probabilities. If $R \leq 0, \mathrm{H}^{1}$ provides a more likely forecast; if $R \geq 0, \mathrm{H}^{0}$ is more likely.

\subsubsection{Simulations}

Assuming a Poisson distribution, each forecasted number of events has a probability of some other number of events occurring consistent with the forecasted number. We use this probability to randomly draw a number of events, for each bin, to create a simulated catalog that is consistent with the forecast. Therefore we are able to obtain a probability distribution of log-likelihood scores for this hypothesis. By comparing the log-likelihood based on the observed events to the distribution of simulated likelihoods, we are able to see how likely it is to observe the real events in the hypothesis. By investigating where the observed log-likelihood falls in the distribution of simulated log-likelihoods, we are able to attach a significance to the observed result. For example, if the observed log-likelihood falls in the lower $5 \%$ of the simulated log-likelihoods, we can reject the hypothesis.

By randomly drawing from the inverse cumulative Poissonian probability density function we obtain the simulated observed events $\hat{\omega}_{i}$ for each given bin. Doing this for all bins, we obtain the total number of simulated events:

$$
\hat{\Omega}^{j}=\left(\begin{array}{c}
\hat{\omega}_{1}^{j} \\
\hat{\omega}_{2}^{j} \\
\vdots \\
\hat{\omega}_{n}^{j}
\end{array}\right), \hat{\omega}_{i}^{j}=\hat{\omega}_{i}^{j}\left(b_{i}\right), b_{i} \in \mathcal{B}
$$

$\Omega_{k}^{j}$ where $j$ denotes which hypothesis forecast was used and $k$ corresponds to an individual simulation run. 


\subsubsection{Consistency test}

In this test we wish to know if we can reject our hypothesis as being consistent with the data. Before we are able to do this, it is up to us to define the significance level $\alpha$ at which we will reject the model. The standard in the literature is typically either 0.05 or 0.01 ; we have chosen to use $\alpha=0.05$.

The significance is defined as the fraction of simulated log-likelihoods that are less than the observed log-likelihood:

$$
\gamma=\frac{\left|\left\{\hat{L}_{k} \mid \hat{L}_{k} \leq L, \hat{L}_{k} \in \mathcal{L}\right\}\right|}{|\mathcal{L}|}
$$

Here $\hat{L}_{k}$ denotes the log-likelihood of the $k^{\text {th }}$ simulation. If $\gamma$ is less than 0.05 we can state that the hypothesis is inconsistent with the data and reject it with with a significance of 0.05 . If $\gamma$ is greater than 0.05 we conclude the hypothesis is consistent with the data based on this this particular measure.

\subsubsection{Ratio Test}

The ratio test allows us to investigate if we can reject the null hypothesis, $H^{0}$,based on the alternative hypothesis, $H^{1}$. To do this, we must first calculate the log-likelihood for each hypothesis based on the observations and then calculate the ratio $R$ of these values:

$$
R=L\left(\Omega \mid \Lambda^{0}\right)-L\left(\Omega \mid \Lambda^{1}\right)
$$

Additionally, we calculate a ratio for every simulated data set, $k$, resulting in a distribution of log-likelihood ratios:

$$
\hat{R}_{k}=L\left(\hat{\Omega}_{k}^{0} \mid \Lambda^{0}\right)-L\left(\hat{\Omega}_{k}^{0} \mid \Lambda^{1}\right)
$$

Significance and rejection are determined using the same method as used in the consistency test and using $\alpha=.05$.

\subsection{The Data}

All earthquake data that we used in the testing comes from the Southern California Earthquake Data Center. The overall $M_{c}$ for the time period we test is claimed to be 1.8 , however the actual value is much higher following large mainshocks. The majority of the events are reported to have horizontal location errors of $\pm 1 \mathrm{~km}$ and depth errors of $\pm 2 \mathrm{~km}$; however occasionally events are report with both errors at greater than $5 \mathrm{~km}$. Magnitude errors are not reported. Location errors of 
less than $5 \mathrm{~km}$ have little effect on our eventual forecast due to the large smoothing radius we use when calculating the seismicity parameters. Better locations would, however, allow us to increase our spatial resolution and potentially create more accurate forecasts. Twenty-four hours following the Landers mainshock, we implement an external fault model. This model is the surface projection of the fault rupture model of Wald and Heaton [1994].

\subsection{Results}

When the testing procedures are coupled with the time-dependent hazard analysis, the calculation becomes very computationally expensive. This expense is compounded when simulations are used. Additionally, time periods where large aftershock sequences are present (1992 - present for southern California) proceed much more slowly than times where few active sequences are present. With the implementation of a computing system designed just for these sorts of problems by RELM, the computation speed should improve markedly. Unfortunately, until that system becomes available, we are limited to performing our test less often and for fewer cumulative years than is desirable.

All tests consisted of daily computed 24 hour rate forecasts that we compared with the observed earthquake numbers and locations for the identical time period. We initiated all tests on January 1 , 1992. However, due to different needs for different tests, i.e., different number of tested parameter sets, some tests were able to run longer than others.

For all tests other than the ratio test against the generic model element, 1,000 simulations were used. Due to time and computational constraints, for the generic test, we used only 100; this is not wholly satisfactory, however the results are strong enough to warrant additional simulations unnecessary.

\subsection{Ratio and Consistency Testing}

The following two tests, measure the consistency of our model with the observed data and the relative consistency of another model as compared to ours.

\subsubsection{Consistency Test Results}

This test was the run from the beginning of 1992 to the end of 1996 using our full model; regionally we tested all of southern California. When calculating a log-likelihood for each 24 hour period and summing all log-likelihood values, the model performed well and could not be rejected. The model was consistent and could not be rejected on days with many earthquakes $(M>4)$ or on days with few to none. 
How to read the S plots: Each plot displays two likelihoodscores:

1) the S-curve is the cumulative distribution of the log-likelihoods based on the simulations. An individual point on the S-curve represents the fraction of overall simulations that obtained a particular log-likelihood score.

2) the log-likelihood of the observed data. All plots are normalized so that the observed log-likelihood is 0.

If the observed log-likelihood ( 0 line) falls in the middle of the simulated log-likelihoods the model is consistent with the data. If the observed is to the left of the simulations (a lower score) it is not among the simulated likelihood scores and is therefore not likely to occur in the model.

The grey bar represents the $5 \%$ rejection area. If any portion of the S-curve touches this line, it may be rejected.

\subsubsection{Background, Generic and Sequence Specific Ratio Tests}

Using the same region as above, but using approximately 3 years of data, we performed three log-likelihood ratio tests where the full composite model was the alternative hypothesis. In each test we used a different null hypothesis: 1) Poissonian background model, 2) generic California model defining an aftershock zone as the area within one Wells and Coppersmith fault length of the mainshock, and 3) a composite model including the generic and sequence specific models (allows for multiple fault models, etc.). The results of the ratio test were consistent in all three tests; the null hypothesis can be rejected with a significance of .05 (fig. 10). On individual days where there where no observed events, the null hypotheses could often not be rejected due to very similar likelihood scores between the models. However on days where events were observed, the alternative hypothesis consistently had significantly higher likelihood scores resulting in the overall rejection of each null hypothesis.

\section{Discussion and Conclusions}

So far our testing has been retrospective, using revised earthquake catalogs. The revised catalogs include corrected locations and magnitudes, and they may take months to be released. Thus our test results are optimistic for two reasons. First, as with any retrospective test, we have the opportunity to make adjustments to the model that would not be possible in a real-time, prospective test. Second, we use corrected data that would not be available in a real time exercise. Location and magnitude errors, and the presence of phantom events that are later edited out of the catalog, could spoil a predictive relationship. We will proceed with a prospective test soon under the RELM project. Should we find a significant improvemet in results based on corrected data, that would 


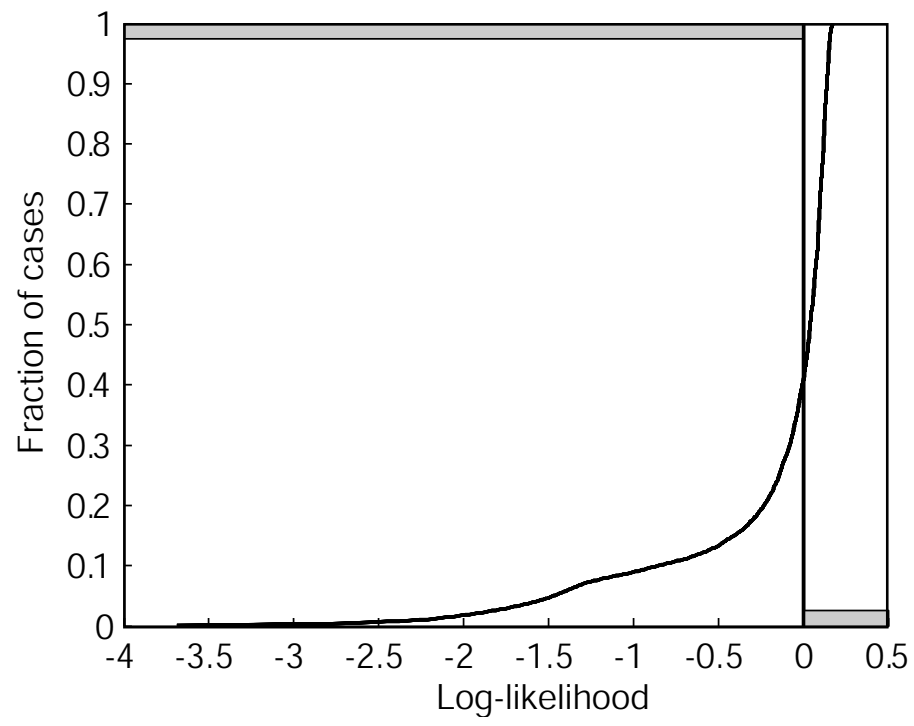

Figure 9: Consistency test results: the sum of all daily log-likelihoods from 1992 through 1996. The model cannot be rejected

illustrate the value of more accurate real-time earthquake location.

For practical reasons discussed in a previous section we have tested forecasted rates of events $(4<M<8)$ and not forecasted ground motions. If all magnitude of events are forecasted equally well, it can be assumed that one test will be equivalent to the other. However, it is possible to foresee a case where a model forecasts the larger and less frequent events better than another model, but forecasts the more frequent smaller events more poorly. This will result in a rejection of the first model when it actually did a better job of forecasting the events that dominate the hazard. This is a problem due to a lack of data; there are simply not enough observed large events to test only based on large events. However, at the lower ground motion levels that we are interested in, a particular magnitude range does not dominate the hazard (as tends to occur in time-independent hazard); therefore the potential for erroneous test results is much less.

The test results show that we now have a valid null hypothesis for use in short term PSHA: our full model including spatial variations. For the short time period we have studied it is clear that the added complexity of spatial variations significantly improve the forecast over more simple models. While on individual days of no activity there is no added benefit from the added complexity, the significant gains in accuracy on days of increased activity means the less complex models can always be rejected.

While we have made a good first step towards a time dependent hazard model, there is always room for improvement. Groups such as RELM are therefore very important to the further development of PSHA as they encourage healthy competition among models and modelers alike. Through 

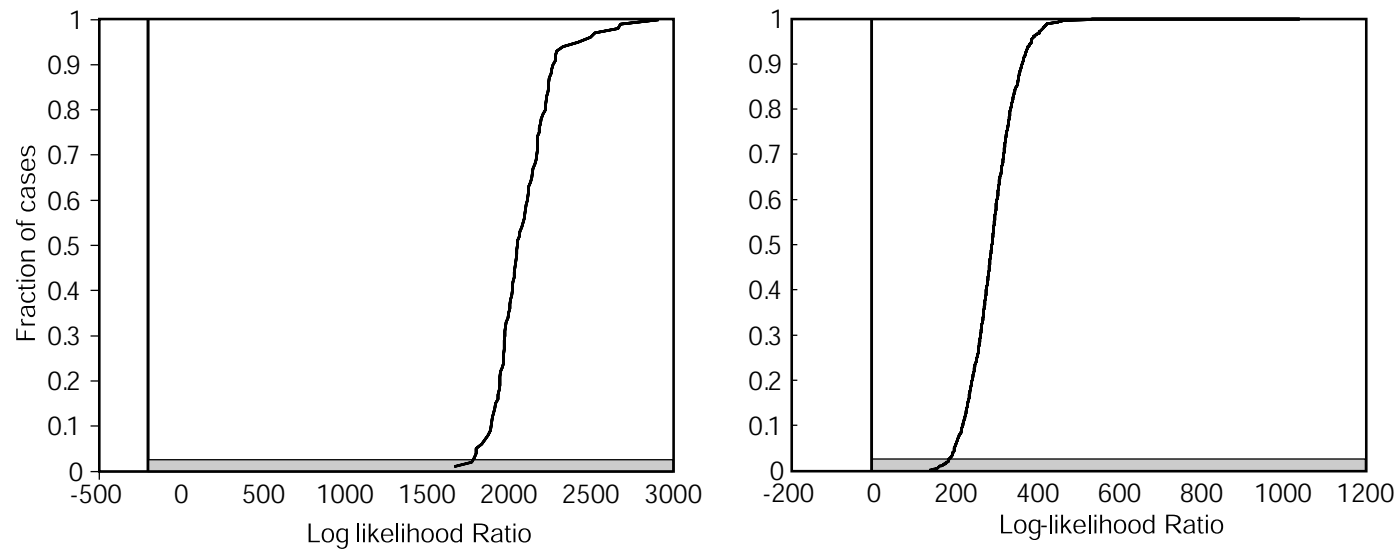

Figure 10: Left: Ratio test for 1992-1994 of generic California model (test 2) against the composite model. Generic model is rejected with a significance of .05. Right: Ratio test for 1992-1995 of composite model excluding any spatially varying information (test 3 ) and the full composite model. The model without spatially varying information can be be rejected with a significance of 05 .

the use of tools that will become available to us in the near future via RELM we will address the questions listed above through much more extensive testing of our own model against itself as well as against other time-dependent models. An important contribution of this will be the organization of real-time prospective testing that is a necessary component of any complete statistical forecast testing routine [Console, 2001].

\section{Acknowledgments}

We thank Ned Field, David Jackson for their helpful reviews and comments; Danijel Schorlemmer for his numerous discussions and input into the development of the processing scheme; and Stan Schwarz and Jan Zeller for their assistance in setting up the necessary IT support to be able to operate in real time.

\section{References}

Aki K., Maximum likelihood estimate of $b$ in the formula $\log N=a-b M$ and its confidence limits, Bull. Earthq. Res. Inst., 43, 237-239, 1965.

Boore, D.M., W.B. Joyner, and T.E. Fumal, Equations for estimating horizontal response spectra and peak acceleration from western North America earthquakes: A summary of recent work, Seismological Research Letters, 68, 128-153, 1997.

Bufe, C.G., and D.J. Varnes, Time-to-failure in the Alaska-Aleutian region: an update, EOS, 77 (F456), 1996. 
Burnham, K.P., and D.R. Anderson, Model Selection and Multimodel Inference A Practical InformationTheoretic Approach, Springer-Verlag, 2002.

Console, R., Testing earthquake forecast hypothesis, Tectonophysics, 338, 261-268, 2001.

Cornell, C.H., Engineering seismic risk analysis, Bulletin of the Seismological Society of America, 58, 1583-1606, 1968.

Enescu, B.a.I., K., Some premonitory phenomena of the 1995 Hygo-ken Nanbu Earthquake: seismicity $b$-value and fractal, Tectonophys., 338(3-4), 297-314, 2001.

Felzer, K., R.E. Abercrombie, and G. Ekstrom, A common origin for aftershocks, foreshocks, and multiplets, submitted Bulletin of the Seismological Society of America, March, 2003.

Field, E.H., Jordan, T.H., and Cornell, C.A., OpenSHA: A developing community-modeling environment for seismic hazard analysis, Seismological Research Letters, 74, 2003.

Frankel, A., C. Mueller, T. Barnhard, D. Perkins, E.V. Leyendecker, N. Dickman, S. Hanson, and M. Hopper, National seismic hazard maps, United States Geological Survey Open-File Report, 96-532, 1996.

Frankel, A., C. Mueller, T. Barnhard, D. Perkins, E.V. Leyendecker, N. Dickman, S. Hanson, and M. Hopper, Seismic hazard maps for California, Nevada and western Arizona/Utah, United States Geological Survey Open-File Report, 97-130, 1997.

Frohlich, C., and S. Davis, Teleseismic $b$-Values: Or, Much Ado about 1.0, Journal of Geophysical Research, 98, 631-644, 1993.

Giardini, D., The Global Seismic Hazard Assessment Program (GSHAP) - 1992/1999, Annali de Geofisica, 42, 957-974, 1999.

Gutenberg, B., and C.F. Richter, Magnitude and energy of earthquakes, Ann. Geofis., 9, 1-15, 1954.

Harris, R., Introduction to special section: Stress triggers, stress shadows, and implications for seismic hazard, Journal of Geophysical Research, 103, 24347-24358, 1998.

Jackson, D., and Y. Kagan, Testable earthquake forecasts for 1999, Seismological Research Letters., 70, 393-403, 1999.

Ji, C., D.J. Wald and D.V. Helmberger, Sip history of the 1999 Hector Mine, California earthquake, Seismological Research Letters, 71, 224, 2000. 
Jolliffe, T., and Stephenson, Forecast verification, a practitioner's guide in atmospheric science, Wiley, 2003.

Kagan, Y.Y., and D. Jackson, Probabilistic forecasting of earthquakes, Geophys. J. Int., 143, 438$453,2000$.

Main, I., Is the reliable prediction of individual earthquakes a realistic goal? Nature online debates, http: //www. nature.com/nature/debates/earthquake,1999.

Matsu'ura, R.S., Precursory quiescence and recovery of aftershock activity before some large aftershocks, Bull. Earthq. Res. Inst., 61, 1-65, 1986.

Michael, A., Testing prediction methods: earthquake clustering versus the Poisson model, Geophysical Research Letters, 24, 15, 2997.

Ogata, Y., Estimation of the parameters in the modified Omori formula for aftershock frequencies by the maximum likelihood procedure, J. Phys. Earth, 31, 115-124, 1983a.

Ogata, Y., Estimation of the parameters in the modified Omori fornula for aftershock frequencies by the maximum likelihood procedure, J. Phys. Earth, 31, 115-124, 1983b.

Ogata, Y., Detection of precursory relative quiescence before great earthquakes through a statistical model, Journal of Geophysical Research, 97, 19845-19871, 1992.

Ogata, Y., Seismicity Analysis through Point-process Modeling: A Review, Pure and Appl. Geophys, 155, 471-507, 1999.

Quitoriano, V., personal communication, 2003.

Reasenberg, P.A., Second-order moment of central California seismicity, 1969-1982, 90, B7, 5479-5495, 1985.

Reasenberg, P.A., Computer Programs ASPAR, GSAS and ENAS and APROB for the Statistical Modeling of Aftershock Sequences and Estimation of Aftershock Hazard, U.S. Geological Survey Open-File Report, 1994.

Reasenberg, P.A., and L.M. Jones, Earthquake hazard after a mainshock in California, Science, 243, 1173-1176, 1989.

Reasenberg, P.A., and L.M. Jones, California aftershock hazard forecast, Science, 247, 345-346, 1990. 
Reasenberg, P.A., and L.M. Jones, Earthquake aftershocks: Update, Science, 265, 1251-1252, 1994.

Reasenberg, P.A., Foreshock occurrence before large earthquakes, Journal of Geophysical Research, 104, 4755-4768, 1999.

Schorlemmer, D., D. D. Jackson, and M.C. Gerstenberger, Earthquake Likelihood Model Testing, in prep, 2003.Stein, R.S., G.C.P. King, and J. Lin, Change in failure stress on the San Andreas and surrounding faults caused by the $1992 \mathrm{M}=7.4$ Landers earthquake, Science, 258, 1328-1332, 1992.

Sieh, et al....

Utsu, T., Ogata, Y., \& Matsu'ura, R. S., The centenary of the Omori formula for a decay law of aftershock activity, Journal of the Physics of the Earth, 43, 1-33, 1995.

Wald, D.J. and Heaton, T.H., Spatial and temporal distribution of slip for the 1992 Landers, California, earthquake. Bulletin of the Seismological Society of America, 84, 668-691, 1994.

Wald, D.J., V. Quitoriano, T.H. Heaton, and H.Kanamori, Relationships between peak ground acceleration, peak ground velocity and Modified Mercalli Intensity in California, Earthquake Spectra, 15, 557-564, 1999a

Wald, D.J., V. Quitoriano, and T.H. Heaton, TriNet "ShakeMaps": Rapid Generation of Instrumental Ground Motion and Intensity Maps for Earthquakes in Southern California, Earthquake Spectra, 15, 537-556, 1999b.

Wells, D.a.C., K. Coppersmith, New emperical relationships among magnitude, rupture length, rupture width, rupture area, and surface displacement, Bulletin of the Seismological Society of America, 84, 974-1002, 1994.

Wiemer, S., Introducing probabilistic aftershock hazard mapping, Geophysical Research Letters, 27, 3405-3408, 2000.

Wiemer, S., Gerstenberger, M., Hauksson, E., Properties of the Aftershock Sequence of the 1999 $M_{w}$ 7.1 Hector Mine Earthquake: Implications for Aftershock Hazard, Bulletin of the Seismological Society of America, 92 (4), 2002.

Wiemer, S., and K. Katsumata, Spatial variability of seismicity parameters in aftershock zones, Journal of Geophysical Research, 104, 13,135-13,151, 1999. 
Wiemer, S., and M. Wyss, Mapping the frequency-magnitude distribution in asperities: An improved technique to calculate recurrence times?, Journal of Geophysical Research, 102, 15115-15128, 1997.

Wiemer, S., and M. Wyss, Minimum magnitude of complete reporting in earthquake catalogs: examples from Alaska, the Western United States, and Japan, Bulletin of the Seismological Society of America, 90, 859-869, 2000.

Wood, H.O., and F. Neumann, Bulletin of the Seismological Society of America, 21 (4), 277-283, 1931.

Wyss, M., Cannot earthquakes be predicted?, Science, 278, 487-488, 1997. 